\title{
Forecasting Waiting Time to Treatment for Emergency Department Patients
}

\author{
Anton Pak ${ }^{* 1}$, Brenda Gannon ${ }^{2,3}$, and Andrew Staib ${ }^{4}$ \\ ${ }^{1}$ Australian Institute of Tropical Health and Medicine, James Cook University, Australia \\ ${ }^{2}$ School of Economics, The University of Queensland, Australia \\ ${ }^{3}$ Centre for the Business and Economics of Health, The University of Queensland, Australia \\ ${ }^{4}$ Emergency Department, Princess Alexandra Hospital, Australia
}

\begin{abstract}
Problem definition. The current systems of reporting waiting time to patients in public emergency departments (EDs) has largely relied on rolling average or median estimators which have limited accuracy. This paper proposes to use the statistical learning algorithms that significantly improve waiting time forecasts.

Practical Relevance. Generating and using a large set of queueing and service flow variables, we provide evidence of the improvement in waiting time accuracy and reduction in prediction errors. In addition to the mean squared prediction error (MSPE) and mean absolute prediction error (MAPE), we advocate to use the percentage of underpredicted observations as patients are more concerned when the actual waiting time exceeds the time forecast rather than vice versa. Provision of the accurate waiting time also helps to improve satisfaction of ED patients.
\end{abstract}

Methodology. The use of the statistical learning methods (ridge, LASSO, random forest) is motivated by their advantages in exploring data connections in flexible ways, identifying relevant predictors, and preventing overfitting of the data. We also use quantile regression to generate time forecasts which may better address the patient's asymmetric perception of underpredicted and overpredicted ED waiting times.

Results. We find robust evidence that the proposed estimators significantly outperform the commonly implemented rolling average. Using queueing and service flow variables together with information on diurnal fluctuations, quantile regression outperforms the best rolling average by $18 \%$ with respect to MSPE and reduces by $42 \%$ the number of patients with large underpredicted waiting times.

Managerial implications. By reporting more accurate waiting times, hospitals may enjoy higher patient satisfaction. We show that to increase the predictive accuracy, a hospital ED may decide to provide predictions to patients registered only during the daytime when the ED operates at full capacity translating to more predictive service rates and the demand for treatments.

Keywords: waiting time; health service; operations management; forecasting.

${ }^{*}$ Correspondence: anton.pak@jcu.edu.au 


\section{Introduction}

Communicating real-time information on waiting times of an emergency department (ED) has been an evolving practice. The innovation of this paper is that it extends a developing agenda of operations research by applying a number of statistical learning methods and constructing a large set of ED predictors.

Waiting times for patients and treatment prioritisation serve as non-price mechanisms through which the ED services addressing health needs of patients are allocated. In countries with publicly funded universal health care systems, like Australia, the price mechanism cannot be exercised even in non-urgent presentations, as the visits to public EDs are covered by the system and patients do not bear any out-of-pocket expenses. In the theoretical section of this paper (see Supplementary material S1), we show that the system is at equilibrium for the low acuity patients when the waiting time reaches the threshold value, and at the margin, the patient is indifferent whether to join the queue or "balk". Apart from the opportunity cost of the waiting time, we also incorporate the intangible effects of the waiting time due to its negative correlation with the patient experience and its influence on the level of anxiety (Mowen et al., 1993).

From an applied perspective, this research focuses on generating accurate waiting time predictions. Waiting time is defined as the time elapsed from the patient's triage time until the start of medical treatment by a doctor, designated treating clinican or treating nurse. Previous research shows that waiting time is one of the key quality metrics in emergency department patient satisfaction (Soremekun et al., 2011; Thompson et al., 1996; Boudreaux and O'Hea, 2004). Early intervention and information communication about the wait time significantly affect the perception of wait time and patient experience (Soremekun et al., 2011). For instance, healthcare networks and individual hospitals in the United States display their current ED waiting times on billboards, websites and in mobile applications; some provinces in Canada and states in Australia have online systems delivering estimated ED waiting times for hospitals located nearby. While potential benefits from publishing ED waiting times include higher patient satisfaction and smoother distribution of patient loads among health providers, there are concerns about the quality of the information reported and how this information is perceived by the patients (American 
College of Emergency Physicians, 2012; Weiner, 2013; Hemaya and Locker, 2012). The current systems of reporting ED waiting times to patients are limited and have largely included rolling average estimates or median historical waiting times.

Using data from a large tertiary hospital in a publicly funded system in Australia we evaluate the performance of quantile and OLS regressions, and statistical learning algorithms such as LASSO, ridge, and random forest and compare the results with the best rolling average predictions. All five estimators significantly outperform the rolling average method by mean squared prediction error (MSPE), mean absolute prediction error (MAPE), and the percentage of underpredicted observations. For example, relative to the rolling average, LASSO reduces the MSPE by $21 \%$ and the quantile regression decreases the number of patients by more than $42 \%$ who experienced waiting time underpredictions exceeding 30 minutes. Furthermore, we show that carefully constructing the variables that represent a complex ED environment with respect to patient queue development, service rates, characteristics of patients and diurnal fluctuations is critical for the estimators to produce high-quality waiting time forecasts.

Recent studies in operations research have implemented various statistical methods to predict the waiting time in emergency department settings. For example, Ang et al. (2015) use Q-LASSO method to prevent overfitting of the waiting time prediction model. The Q-LASSO estimator reports higher accuracy than the rolling average estimator. Relative to Ang et al. (2015) study, our sample of patients for whom we generate waiting time predictions is slightly different and includes only low acuity patients located in the ED waiting room. The prediction of the waiting time to treatment potentially benefits these patients the most, because they are more likely to experience longer waiting time to treatment. Knowledge of approximately how long they need to wait in the ED waiting room before the treatment commences may help to reduce their anxiety and decrease the number of patients "who leave without being seen" (LWBS).

Another approach to predict the waiting time in ED was proposed by Sun et al. (2012) in which the authors test quantile regression model using the data from a Singaporean hospital. One of the main limitations of that study is the data availability that does not allow authors to construct a rich set of predictors, thus limiting the improvement in the accuracy of the estimators. In addition, while the authors focus on the results from 
the median and $95^{\text {th }}$ quantile for quantile regression, we provide evidence that selecting high quantile values results in not only smaller number of underpredicted observations, but also larger prediction errors due to increasing overprediction cases. In our study we use cross validation based on this trade-off to select the quantile threshold for quantile regression predictions.

ED waiting time can also be influenced by the changes in prioritisation within triage systems (Ding et al., 2019) and proactive diversion policies (Xu and Chan, 2016). Using simulation analysis, $\mathrm{Xu}$ and Chan (2016) show that such policies can reduce delays by up to $15 \%$. Furthermore, Batt and Terwiesch (2015) study patient behaviour with regard to observable changes in the waiting time. In particular, they propose a framework to study the phenomenon of queue abandonment by patients. Dong et al. (2018) provide evidence through simulation analysis that ED patients use online ED waiting times for two nearby hospitals to make a decision about which hospital ED to attend. This innovative approach of posting accurate waiting time to treatment for less urgent patients for multiple EDs, especially, in metropolitan areas may increase the social welfare and reduce the average waiting time for all the hospitals and smooth the arrivals of the patients across the ED network.

This paper is relevant to the current literature from two perspectives. First, we examine whether there is an improvement in prediction accuracy by implementing quantile regression and statistical learning algorithms on a large set of predictors using comprehensive electronic patient records from the ED information system. Although some hospitals in Australia implemented the simple prediction methods, such as rolling average, the use of advanced statistical learning algorithms may have high potential for accuracy improvement. And second, we extend the literature by forecasting ED waiting time for low acuity patients allocated to the ED waiting room. We provide strong arguments for differentiating the low acuity patients into waiting room and non-waiting room patients for the purpose of waiting time prediction. Furthermore, we examine the external validity of existing prediction methods using the data from a large tertiary hospital in Australia. 


\section{Clinical setting}

To provide an accurate estimate of the waiting time for low acuity ED patients, we apply a range of statistical methods using routinely collected administrative data from a single hospital, Princess Alexandra Hospital (PAH), for the period of 2 years (1 January 2016 to 31 December 2017). PAH is a large public tertiary health care centre located in Brisbane Metro Area in the state of Queensland, Australia and provides care in all major adult specialties except maternity. PAH has been at the forefront of hospital digital transformation in Australia and one of the first hospitals that implemented a new information system in ED, Cerner FirstNet, in a fully integrated manner (Sullivan et al., 2016).

Cerner FirstNet is our primary source of data. It provides the detailed time and clinical information about the patient's journey in ED (arrival $\rightarrow$ throughput $\rightarrow$ departure) that is necessary for accurate prediction when applying statistical learning algorithms. Data for each presentation is recorded at the start of the registration and triage process and completed at the time of ED departure or hospital admission. Annually the ED treats around 60,000 patients and is staffed with specialist emergency physicians and nurses 24 hours a day. Our initial sample contains information on 122,716 ED presentations.

We define low acuity patients as patients with triage categories 3,4 , and 5 . These patients represent individuals with lower priority for medical treatment than more urgent ones (triage categories 1 and 2) due to the acuity of their condition. Out of 122,716 ED presentations in our sample, 94,284 were of low acuity and 28,432 were defined as high acuity patients having triage categories 1 and 2 . In Figure 1, we show that there were clear differences in waiting time during the day between high and low acuity patients. The average waiting time for high acuity patients was relatively constant during the day and they were treated quickly after arrival. The resuscitation (triage 1) patients received medical treatment almost immediately and triage 2 patients on average waited no longer than 10 minutes. On the other hand, there were large fluctuations in waiting time for triage 3,4 , and 5 patients with no clear indication of a difference in average wait times by triage category in this group. This reflects the departmental workflow where low acuity patients are generally seen in order of arrival rather than by triage category.

In Figure 2 we describe the simple ED patient flow for low acuity cases. Upon 


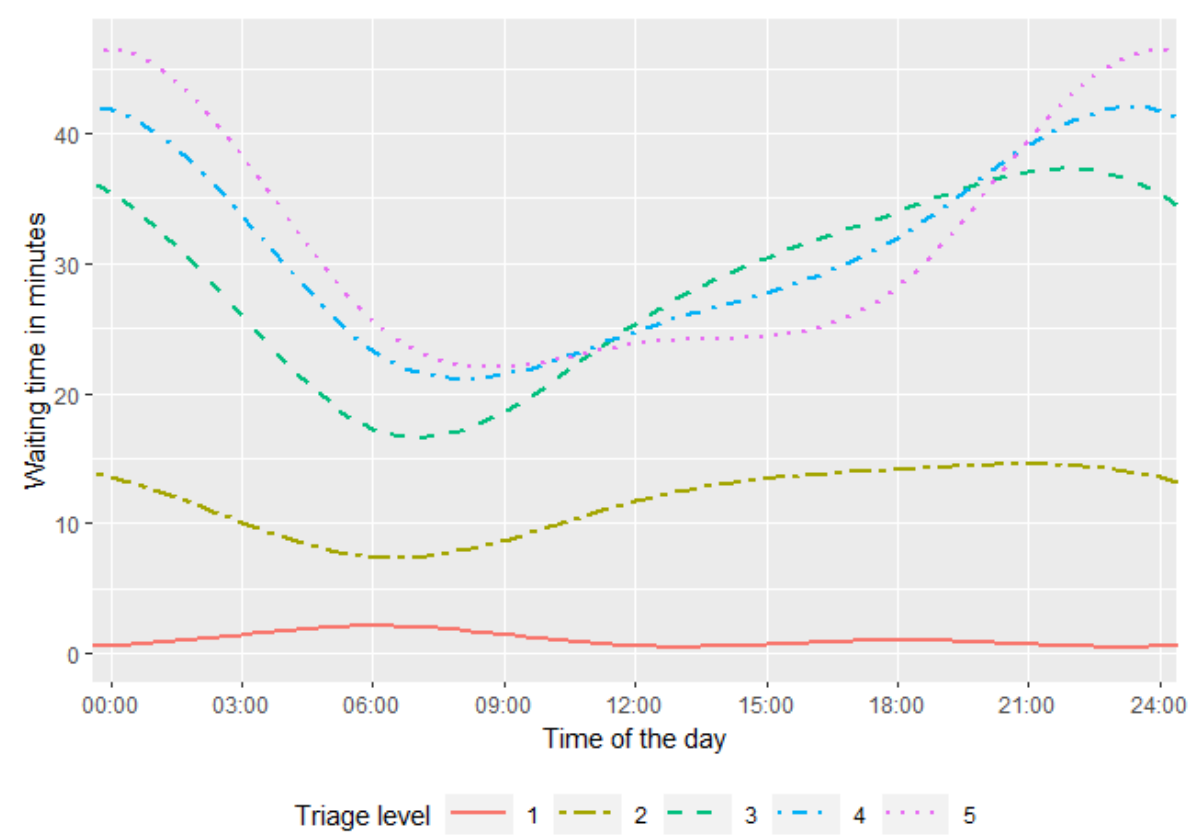

Figure 1: Average waiting time by triage level

arrival (stage 1), a low acuity patient may experience a queue (stage 2) to see a triage nurse (stage 3) who records some basic identification details, a presenting complaint, describes the nature of the visit, and assigns a triage level and a location after triage. The patient then proceeds to the registration window (stage 4), where further personal information (e.g. address, occupation) is collected and the electronic medical record registration is completed. After the registration process, the patient, if not called in for treatment immediately, proceeds to the designated waiting area. The allocation of patients to the waiting room (stage 5a), ambulatory care unit (ACU) (stage 5b), mental health waiting room (stage 5c) or acute bed area (stage 5d) depends on multiple factors, such as a patient's health condition, occupancy rates and bed availability, staff availability, demand predictions and time of the day. A patient needs to wait in a designated waiting area until being called in to see a clinician or a treating nurse may come out to the waiting area and take vital sign measures and periodically check on the patient while he is waiting to be called in for treatment.

In general, patients in different ED areas are treated by different ED teams. For example, ED PAH is equipped with $\mathrm{ACU}$ - that treats patients who are suitable for care in ambulatory environment. These patients tend to be of lower acuity with a lower admission rate than non-ambulatory patients. This unit facilitates same day discharge of patients 


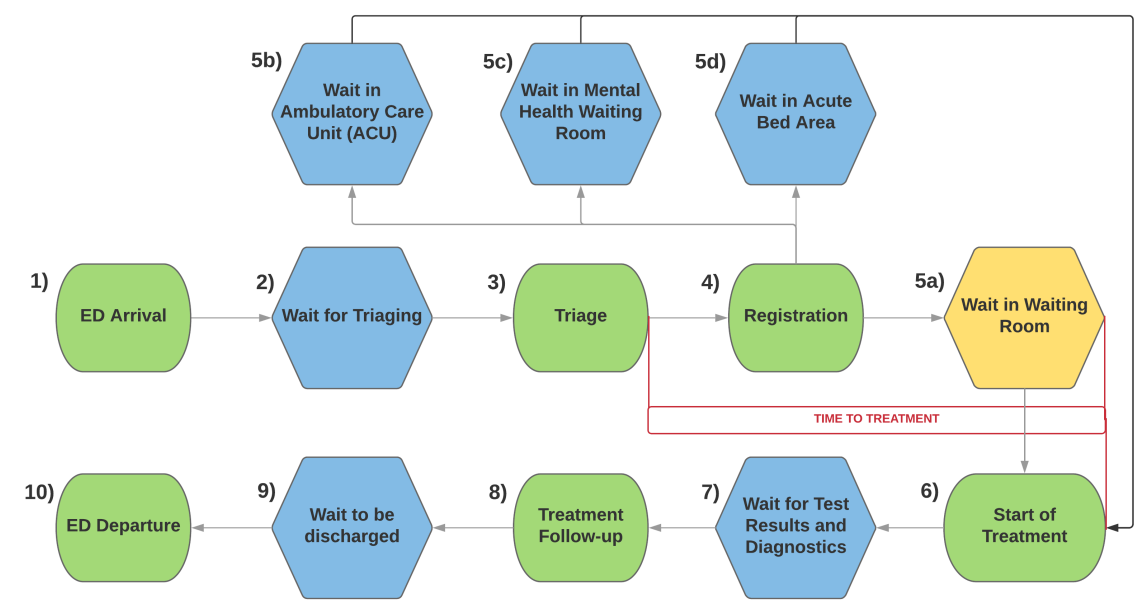

Figure 2: Simple ED patient flow

and many of the waiting room low acuity patients are managed in ACU, although the unit does not operate at night and these patients are treated in other areas depending on bed availability during the night hours.

ED PAH also operates a Short Stay Unit (SSU) that is specifically designed for short term treatment (often completed within 24 hours). This is an inpatient unit under ED management providing health services to patients that were initially assessed, triaged and treated in the ED. Most often patients with selected medical conditions that require further observation, assessment, and reassessment but are unlikely to require a prolonged admission to hospital are assigned to SSU.

\subsection{Focusing on waiting room patients}

In this research we design the forecasting models to predict the waiting time to treatment for low acuity patients who have been allocated to the waiting room (stage $5 \mathrm{a}$ in Figure 2) after the triage stage. In Figure 3 we report a clear difference in waiting times for patients who have been allocated to the waiting room and the other non-ambulatory waiting areas. Overall, in Figure 3 we show not only that waiting room patients exhibited significantly larger waiting times on average, but also that there was a limited variation in the waiting times between triage categories 3,4 , and 5 , which makes it more reasonable to combine them together for the purpose of waiting time prediction. Also regardless of the low acuity triage levels, the mean time-to-treatment for patients in the waiting room 
is around 28 minutes, while non-waiting room patients experience the wait time of 21 minutes across triage categories 3,4 , and 5 .

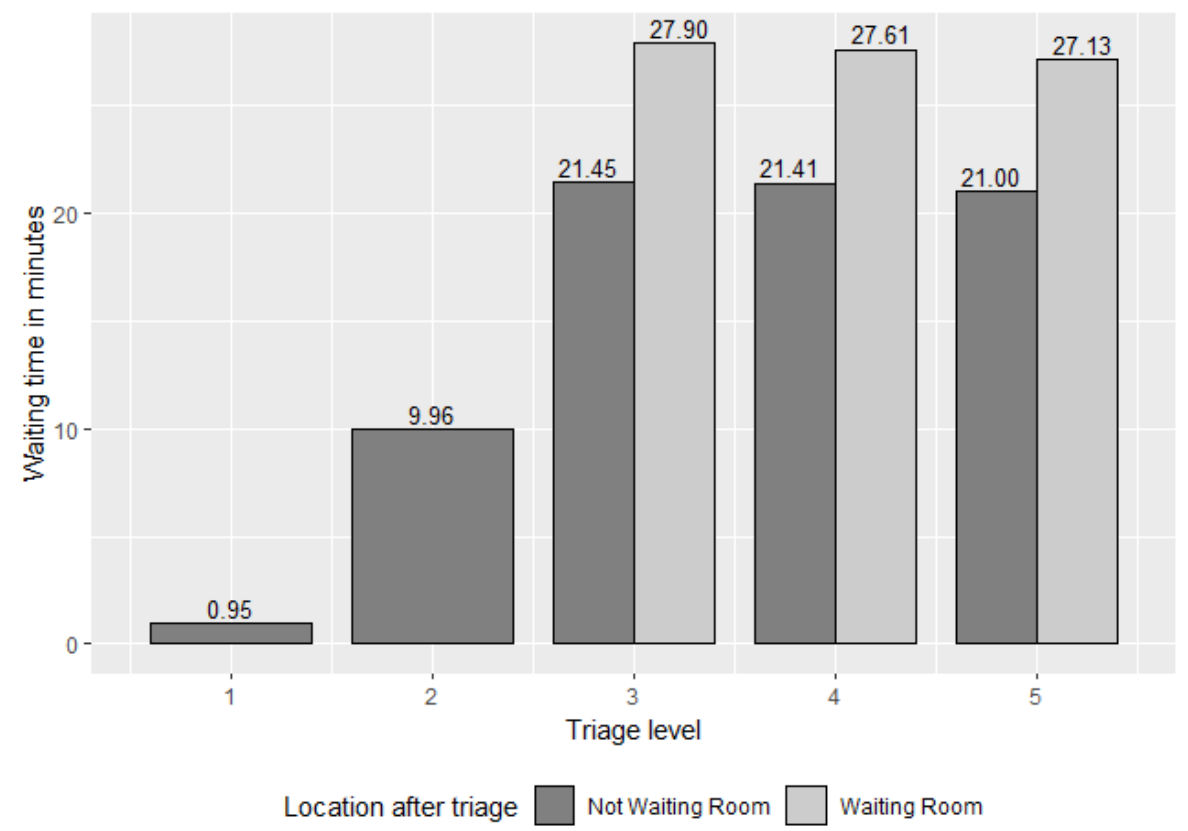

Figure 3: Average waiting time by triage and waiting room status

Health reasons also play a role in assigning the location after triage. In general, patients who are allocated to the waiting room do not require close monitoring or medical attention while waiting, in comparison to patients allocated to the ACU, acute bed area or mental health waiting room. Furthermore, we focus on waiting room patients because they may benefit the most from the waiting time prediction. In the current setting with no delay announcement system, it is difficult for patients to estimate the approximate waiting time, as they are not aware of their own and other patients' triage status who are waiting in the ED waiting room. Waiting patients get treated generally, but not necessarily, on the first-come-first-serve basis following the triage order prioritisation. As for the supply side, patients awaiting treatment in the waiting room receive no information about bed occupancy, number of patients currently being treated, queue for diagnostic procedures and other supply side characteristics that influence the rate of servicing patients. On the contrary, this information may be visibly available to the patients who are waiting in the acute bed or ACU areas - which helps patients' perception of the waiting time, as patients observe the busyness of the department and resource availability.

It also takes different levels of effort for patients to leave the ED if they decide not 
to wait for treatment. In ED PAH and many other public EDs the waiting room is located next to the ED entrance and there are no barriers for patients to leave the ED once their waiting time becomes larger than their willingness to wait threshold. On the other hand, patients in the ACU and acute bed areas are inside ED treatment areas and behind the doors. Even if the waiting time is long, psychologically it may be harder to leave the ED for those patients.

\section{Methodology}

For building the prediction model for the waiting time for less urgent patients, we use quantile regression, OLS, and statistical learning algorithms such as random forest, ridge, and LASSO. There is a gap in the literature on predicting waiting time and measuring the accuracy of the estimators in the ED setting. Hemaya and Locker (2012) implemented OLS to generate waiting time predictions for patients, but raised concerns about the degree of inaccuracy that the patient might find satisfactory. Ang et al. (2015) provide some evidence that LASSO estimator outperforms rolling averages, quantile regression, and fluid models and they focus on the importance of predictors' selection. The use of the statistical learning methods (ridge, LASSO, random forest) is motivated by their advantages in exploring data connections in flexible ways, identifying relevant predictors and preventing overfitting of the data. We also contribute to the literature by analysing the performance of the random forest algorithm in the context of ED waiting time predictions. In addition to the statistical learning methods, we use quantile regression to generate time forecasts which may better address the patient's asymmetric perception of underpredicted and overpredicted ED waiting times.

We follow the established statistical practice for predictive models, and divide the sample into two parts: 1) training sample, which is used to construct the prediction algorithms $(\Phi)$; and 2) testing sample, which we use to test and compare the predictive accuracy of the algorithms $(\Psi)$. To build the prediction models, we define $X$ as the vector of random variables $\left(X_{1}, X_{2}, \ldots, X_{p}\right)$ denoting input variables, often referred as model features in statistical learning literature. Random variable $W T$ represents the output variable denoting the wating time to treatment. Generating waiting time predictions 
using the input variables is expressed by the prediction model, $\Omega$, trained on the sample $\Phi=(X, W T)$. The objective function of the models is to produce predictions $\left(\Omega_{\Psi}\right)$ that are close to the actual values of $y_{\Psi}$, hence minimising the expected prediction error. This is defined as follows:

$$
\operatorname{Err}_{\Psi}(\Omega)=\frac{1}{N_{\Psi}} \sum_{\left(x_{i}, W T_{i}\right) \in \Psi} \mathrm{L}\left(W T_{i}, \Omega_{\Phi}\left(x_{i}\right)\right)
$$

where $\mathrm{L}\left(W T_{i}, \Omega_{\Phi}\left(x_{i}\right)\right)$ is the prediction error between the actual waiting time from the testing sample and the waiting time prediction generated by the model $\Omega_{\Phi}$.

Using the testing sample we evaluate the accuracy of the prediction by calculating MSPE, MAPE, and the percentage of underpredicted observations. MSPE uses the squared error loss function, which provides heavier penalty for larger differences between the actual and predicted waiting times. This loss function is denoted as follows:

$$
\operatorname{Err}_{\Psi}(\Omega)=\frac{1}{N_{\Psi}} \sum_{\left(x_{i}, W T_{i}\right) \in \Psi}\left(W T_{i}-\Omega_{\Phi}\left(x_{i}\right)\right)^{2}
$$

MAPE as a criterion complements MSPE well, as it is less sensitive to outliers, and it helps to validate the results for the model selection. MAPE is calculated by:

$$
\operatorname{Err}_{\Psi}(\Omega)=\frac{1}{N_{\Psi}} \sum_{\left(x_{i}, W T_{i}\right) \in \Psi}\left|W T_{i}-\Omega_{\Phi}\left(x_{i}\right)\right|
$$

The third criterion we use to evaluate the performance of the prediction models is the percentage of underpredicted observations, defined as the number of underestimated waiting time predictions by more than 30 minutes divided by the total number of observations in the testing sample, and then multiplied by 100. This performance measure is important from the hospital's practical perspective, because the waiting patients are more dissatisfied when not treated after the predicted waiting time elapsed forgetting that the waiting time prediction is only indicative and exhibit a non-binding commitment (Ang et al., 2015). The percentage of underpredicted observations is represented by:

$$
\operatorname{Err}_{\Psi}(\Omega)=\left[\frac{1}{N_{\Psi}} \sum_{\left(x_{i}, W T_{i}\right) \in \Psi} \mathbf{I}\left\{W T_{i}>\Omega_{\Phi}\left(x_{i}\right)+30\right\}\right] * 100 \%
$$


where $\mathbf{I}\{\cdot\}$ denotes an indicator function.

The statistical learning methods (ridge, LASSO, random forest) and quantile regression may have high potential for accuracy improvement in comparison to the popular rolling average method. We use the rolling average as a reference model as the predictions from this estimator is most commonly reported in Australian ED health services. To calculate the rolling average waiting time estimate we use:

$$
\widehat{W T_{i t}}= \begin{cases}0, & \text { for } k=0 \\ \frac{1}{K} \sum_{k=1}^{K} W T_{k,[t-h, t]}, & \text { for } k \geq 1\end{cases}
$$

where $t$ represents the time when a current patient $i$ is triaged and for whom the prediction is generated; $h$ is the number of hours determining the time window; $k$ defines a patient who was waiting and got into treatment during the time window $[t-h, t]$, so that the patient's waiting time is known and defined by $W T_{k,[t-h, t]}$.

Although the rolling average is computationally inexpensive to implement and requires only the information about the waiting time of patients who got into treatment, the method does not take into account the capacity constraints, service flow characteristics, high acuity arrivals and, most importantly, the demand predictions. Using only historical waiting times in a complex ED environment limits the accuracy of the rolling average estimator making hospital EDs reluctant to provide waiting time estimates due to the high over- and under-prediction errors.

\subsection{OLS, ridge, and LASSO regressions}

To predict the waiting time to treatment for low acuity patients we use a large set of predictors. Using the standard least squares representation with $W T_{i}$ - waiting time for an individual $i$, and a vector $x_{i}$ being a set of predictors and attributes related to $i^{\text {th }}$ individual, we describe the model as:

$$
W T_{i}=\beta_{0}+\sum_{p=1}^{P} \beta_{p} x_{i p}+\epsilon_{i}
$$


Ridge and LASSO regressions apply the least squares method to fit a linear model choosing a subset of predictors determined by the regularization term. The method selects regression coefficients $\hat{\beta}$ to solve the loss function defined by:

$$
\min _{\beta \in R^{p}} \frac{1}{N} \sum_{i=1}^{N}\left(W T_{i}-\beta_{0}-\sum_{p=1}^{P} \beta_{p} x_{i p}\right)^{2}+\lambda\left[(1-\alpha) \sum_{p=1}^{P} \beta_{p}^{2}+\alpha \sum_{p=1}^{P}\left|\beta_{p}\right|\right]
$$

This minimisation function consists of the standard least squares sum of squared residuals (SSR) and the shrinkage component (i.e. regularization term) which differentiates the minimising quantity for ridge $(\alpha=0)$ and LASSO $(\alpha=1)$ regressions. The tuning parameter $\lambda$ determines the importance of the shrinkage component in the minimisation problem. If we impose a restriction of $\lambda=0$, then the estimator becomes OLS. At the other extreme, as $\lambda \rightarrow \infty$ the impact of the shrinkage penalty becomes dominant in the estimator and forces the estimated parameters $\beta$ to be close to 0 . The usefulness of introducing the penalty function lies in the bias-variance trade-off. By using regularization term (defined by the penalty function), we aim to prevent overfitting of the data and provide a better compromise between bias and variance of the estimated parameters. An increase in the tuning parameter $\lambda$ leads to the decrease in the model fit (equivalent to the increase in the bias), but at the same time reduces the variance of the parameters. Selecting $\lambda$ thus becomes critical in order to find the right balance between bias and variance; and we apply $k$-fold cross validation method to choose $\lambda$. The cross validation process is explained in the Supplementary material S2.

\subsection{Random forest}

While OLS, ridge, and LASSO statistical learning methods assume a linear structure between the covariates and the outcome, tree-based estimators are non-parametric and provide an effective way of uncovering complex data connections. They are robust to outliers and like LASSO and ridge implement feature selection algorithm, which ranks the variables according to their importance and minimises the influence of irrelevant factors in explaining the outcome. We first introduce the construction of decision trees, as they are the basis for the random forest method.

Our data consists of a response variable $(W T)$ and input variables $X$ defined as the 
vector of $p$ random variables $\left(X_{1}, X_{2}, \ldots, X_{P}\right)$. The algorithm partitions the input space into $S$ disjoint regions $\left(R_{1}, R_{2}, \ldots, R_{S}\right)$ and then assigns constant prediction $\widehat{W T_{R_{s}}}$ to all observations that are mapped within each region $s$. If we adopt the squared error loss function, then the objective of a single decision tree is to split the input space $X$ in such a way that minimises the function:

$$
\begin{gathered}
\sum_{s=1}^{S} \sum_{i \in R_{s}}\left(W T_{i}-\widehat{W T_{R_{s}}}\right)^{2} \\
\widehat{W T_{R_{s}}}=\frac{1}{N_{R_{s}}} \sum_{\left(W T_{i}, x_{i}\right) \in R_{s}} W T_{i}
\end{gathered}
$$

where $\widehat{W T_{R_{s}}}$ is the waiting time mean response using training sample for the $s^{\text {th }}$ region of predictors space. We explain the partition procedure in Supplementary material S2.

Random forest algorithm provides great benefits to variance reduction by growing multiple trees from bootstrapped samples and averaging the results. The algorithm of the random forest includes the following steps:

1. Generate a bootstrap resampled dataset from the training data;

2. Using bootstrapped data build a tree with randomly chosen $k$ out of $p$ predictors before each split. Following Hastie et al. (2009, p. 589 ), we choose the number of predictors using $k \approx \sqrt{p}$;

3. Repeat steps 1 and 2 for selected number of trees (we use $T=100$ );

4. Average the predictions from the generated random forest trees for each testing observation: $\widehat{W T}_{i}=\frac{1}{100} \sum_{t=1}^{100} \widehat{W T_{i t}}$, where $\widehat{W T_{i t}}$ specifies a waiting time prediction for an individual $i$ using a decision tree $t$.

Selecting $k$ predictors randomly at each split allows to decorrelate the trees and hence reduces the variance of the predictions and prevents overfitting of the data. Random forest estimator often performs well and achieves high predictive accuracy, as the method does not impose any distributional assumptions on the data and captures well the nonlinear relationship between the set of potential predictors and the dependent variable, waiting time in our case. 


\subsection{Quantile regression}

Quantile regression is a more flexible estimator for modelling data with heterogeneous conditional distribution, and it provides richer characterisation of the data showing the effects of independent variables across the spectrum of the dependent variable. By selecting a quantile value, $q$, and estimating the conditional quantile regression function, we can generate out-of-sample prediction for the $q^{\text {th }}$ quantile waiting time. Since we are more concerned with underpredictions (actual waiting time is larger than predicted waiting time), the quantile regression may mitigate the problem if we select higher than median quantile values. In comparison to the least squares methods, the quantile regression estimator is less sensitive to outliers, which is advantageous as in the complex ED environment there are some high values of the actual waiting time.

After defining the conditional quantile regression function, $Q_{q}(y \mid x)$, where the conditional quantile is taken to be linear in covariates $x$, the $q^{\text {th }}$ quantile regression estimator minimises the following function over parameters $\beta_{q}$ :

$$
\min _{\beta_{q}} \sum_{i: W T_{i} \geq x_{i}^{\prime} \beta_{q}}\left(q\left|W T_{i}-x_{i}^{\prime} \beta_{q}\right|\right)+\sum_{i: W T_{i}<x_{i}^{\prime} \beta_{q}}\left((1-q)\left|W T_{i}-x_{i}^{\prime} \beta_{q}\right|\right)
$$

By using quantile regression we focus on the tails of the waiting time distribution, in particular on the right tail. We select the quantile that is above the median to provide higher weights to the positive residuals resulting in a lower number of underpredicted observations.

\section{Description of predictors}

The selection of variables is one of the key components for the prediction models. We construct queueing and service flow variables to account for ED activity, time, and weather variables to control for demand fluctuations during different time periods and weather conditions. We also include patient characteristics such as triage category and patient's age that potentially may influence the heterogeneity in waiting time forecast.

The waiting time of low acuity patients allocated to the waiting room largely depends on the current ED activity. Queues are built by patient arrivals, ED activity, and 


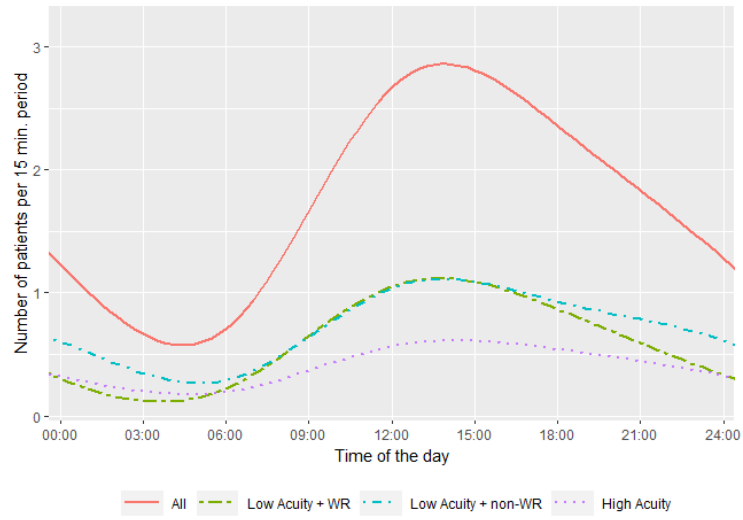

Figure 4: Average arrivals of patients

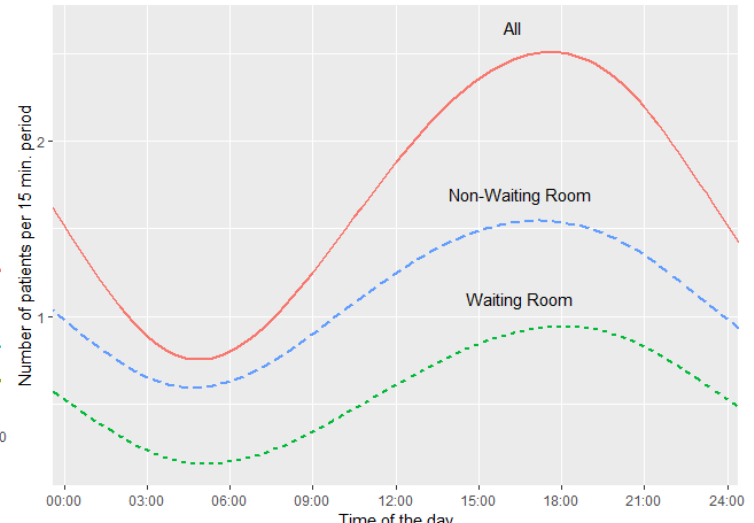

Figure 5: Average number of departed patients

service rate of patients, so we investigate how the queueing and service flow measures develop during the day. In Figure 4, we use the rate of patient arrivals by triage in the previous 15-minute non-overlapping period to account for the ED services demand. It is not surprising that the arrivals of patients is not consistent during the day with more patients arriving during morning, afternoon, and early evening when population is generally more physically active and more likely to seek help in the ED. For the periods with higher ED arrival rates $(6 \mathrm{am}-6 \mathrm{pm})$ there are no visible differences in the average arrival rates between patients allocated to the waiting room and other waiting areas. Furthermore, we calculate the size of the queue of the waiting patients for each arriving patient using their triage and start of treatment times.

In Figure 5 the average number of departed patients shows a similar pattern of ED arrivals but with an approximately 4 hour lag, which resonates well with the National Emergency Access Target (NEAT) that promotes $90 \%$ of ED cases be completed (either with patient admission or discharge) within four hours of presentation (Queensland Audit Office, 2014).

In addition to the ED arrivals and departures measures, the number of patients starting treatment in the past 15 minutes presents a service flow measure. Figure 6 resembles the pattern in Figure 4 (ED arrivals) reporting that most of the patients do not experience a long waiting time between presentation and start of the treatment.

Our ED activity variables also include the number of high acuity and low acuity patients in the treatment. High acuity patients require more medical attention, especially during the time of arrival, when the ED resources may be diverted to deal with complex 


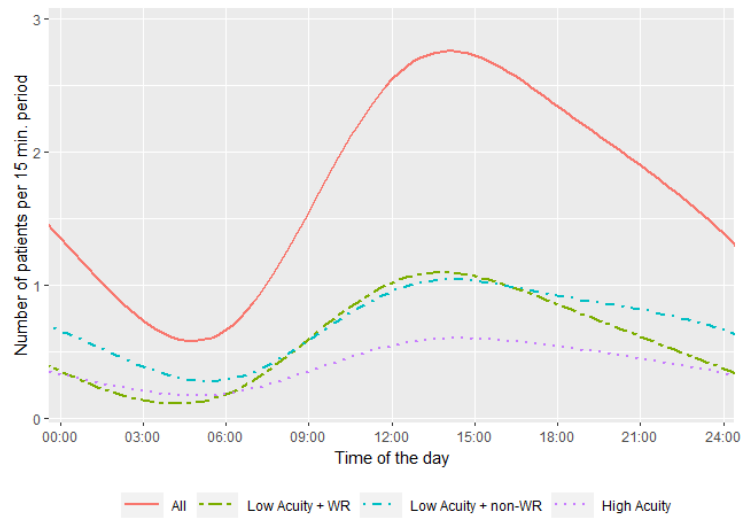

Figure 6: Average number of patients starting treatment

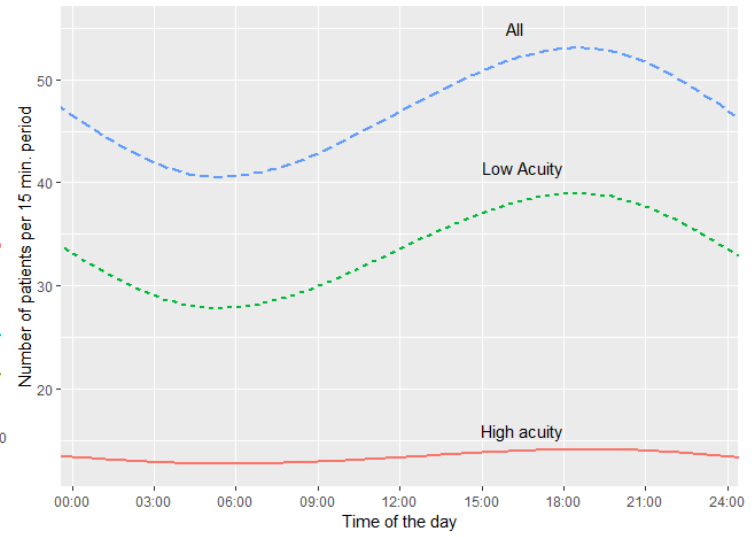

Figure 7: Average number of patients in the treatment

cases, hence increasing the waiting time for other patients awaiting treatment. In Figure 7 , we report that during the day the number of high acuity patients in the treatment remains stable, while the number of low acuity treated patients fluctuates and follows the demand from ED arrivals with some lag due to the waiting time.

The ED activity, as we have shown previously, highly fluctuates during the day. To accommodate these daily fluctuations, we include diurnal 15-minute non-overlapping period controls dependent on the patient's ED triage time. Previous literature has provided evidence that the demand for ED services varies during the days of the week, especially for the low acuity cases. This is largely due to the limited availability of primary care services during the weekend and after-hours periods (Dolton and Pathania, 2016; Bruni et al., 2016). In Table 1 we show that the largest number of low acuity ED presentations occurs on Mondays, Tuesdays, and Sundays. We capture these daily fluctuations in demand by including days of the week dummy variables in the set of predictors.

Table 1: ED presentations by day of the week

\begin{tabular}{cccccccc} 
& Monday & Tuesday & Wednesday & Thursday & Friday & Saturday & Sunday \\
\hline ED arrivals & 6172 & 5847 & 5421 & 5453 & 5496 & 5329 & 5577
\end{tabular}

Seasonality and holiday periods may also affect ED crowding. For example, influenza follows a seasonal pattern and during the epidemics we expect more ED crowding. On the other hand, holiday periods may be associated with more people participating in outdoor and physical activities potentially leading to more emergency accidents. Similarly, weather variables may influence how much people spend time outside and engage in 
physical exercises. We include average temperature, atmospheric pressure, humidity level and presence of rain to control their effects on the ED demand. Additionally, weather inconveniences may influence the decision of low acuity patients (in particular, triage 5) to attend an ED.

Furthermore, some patient and presentation-specific characteristics may influence the waiting time of patients. Together with the dummy variables for the patient triage statuses, we specify whether a patient's presentation was mental-health related and whether a patient arrived by an ambulance or police transport. Patients presenting for assessment of mental health complaints are assessed and managed by a different clinical team than the remaining patient population. The mental health clinicians are also responsible for managing mental health patients in other areas of the department, and as such can be subject to competing, more clinically urgent priorities. The full list of predictors and their description are available in Table 2.

\section{Results}

Out of 39,295 low acuity ED patients allocated to the waiting room we use the first $80 \%$ of observations or 31,436 for training the forecasting models and the last $20 \%$ or 7859 observations for generating out-of-sample predictions and testing which models perform the best. To evaluate the models we use three criteria: MSPE, MAPE, and the percentage of underpredicted observations.

For quantile regression estimator we select the $q^{\text {th }}$ quantile based on the combination of out-of-sample MSPE and percentage of underpredicted observations criteria. Figure 8 shows the criteria results under different specifications of the predictors set by quantile. Not surprisingly, there is a trade-off between the two criteria at the upper quantiles. As the quantile value increases, the quantile regression places higher weights on positive residuals leading to reduction in the percentage of underpredicted observations.

For example, for the $95^{\text {th }}$ quantile the percentage of underpredicted waiting time by more than 30 minutes is less than 1\%. Quantile regression predictions are also easy to interpret and explain to patients. By generating waiting time predictions for $95^{\text {th }}$ quantile, we can expect that no more than $5 \%$ of the patients will wait longer than the predicted waiting 
Table 2: Description of predictors

\begin{tabular}{|c|c|c|}
\hline Predictor & Description & $\begin{array}{l}\text { Number } \\
\text { of Variables }\end{array}$ \\
\hline \multicolumn{3}{|c|}{ Queueing / service flow variables $(Q)$} \\
\hline $\begin{array}{l}\text { c.arrivals.t1 } \\
(2,3,4,5)\end{array}$ & $\begin{array}{l}\text { count for arrivals of triage } 1(2,3,4,5) \text { patients to the ED in the previous } \\
15 \text {-minute interval; }\end{array}$ & 5 \\
\hline c.left.wait & $\begin{array}{l}\text { count for the number of waiting room patients who departed in the previous } \\
15 \text { minutes }\end{array}$ & 1 \\
\hline c.left.nowait & $\begin{array}{l}\text { count for the non-number of waiting room patients who departed in the pre- } \\
\text { vious } 15 \text { minutes }\end{array}$ & 1 \\
\hline c.wait & $\begin{array}{l}\text { count for waiting patients who have not yet been seen by a doctor (ED con- } \\
\text { sultant or treating clinician) or treating nurse by the time a current patient is } \\
\text { triaged }\end{array}$ & 1 \\
\hline c.treat.wait & $\begin{array}{l}\text { count for the number of wait patients who started treatment and now in the } \\
\text { treatment }\end{array}$ & 1 \\
\hline c.treat.nowait & $\begin{array}{l}\text { count variable for the number of non-waiting room patients who started treat- } \\
\text { ment and now in the treatment }\end{array}$ & 1 \\
\hline $\begin{array}{l}\text { c.treat.triage1 } \\
(2)\end{array}$ & $\begin{array}{l}\text { count variable for the number of triage } 1(2) \text { patients in the treatment by the } \\
\text { time a current patient is triaged. Note: time for starting treatment is the same } \\
\text { as triage time for those patients }\end{array}$ & 2 \\
\hline $\begin{array}{l}\text { c.treat.triage } 3 \\
(4,5)\end{array}$ & $\begin{array}{l}\text { count variable for the number of triage } 3(4,5) \text { patients in the treatment by } \\
\text { the time a current patient is triaged. Note: time for starting treatment is the } \\
\text { same as triage time for patients who did not wait in the waiting room }\end{array}$ & 3 \\
\hline c.lwbs & $\begin{array}{l}\text { count for the number of patients who did not wait for treatment in the last } 4 \\
\text { hours before a current patient's triage time }\end{array}$ & 1 \\
\hline \multicolumn{3}{|c|}{ Rolling average variables $(\mathrm{RA})$} \\
\hline c.3hrs & $\begin{array}{l}\text { count for the number of patients who were waiting and got in the treatment } \\
\text { in the last } 3 \text { hours from the current patient triage time } \\
\text { rolling average time variable for the average waiting time for patients who were }\end{array}$ & 1 \\
\hline t.avg_3hrs & $\begin{array}{l}\text { waiting and got in the treatment in the last } 3 \text { hours from the current patient } \\
\text { triage time }\end{array}$ & 1 \\
\hline amcare & $\begin{array}{l}\text { Ambulatory care unit }(\boldsymbol{A}) \\
\text { dummy variable specifying whether the ambulatory care unit was opened at } \\
\text { the time of presentation. ACU working hours: } 9: 00-23: 30 \\
\text { Time variables }(\boldsymbol{T})\end{array}$ & 1 \\
\hline t.15.1 (2-95) & dummy variable for the first (00:01 - 00:15) diurnal 15-minute interval. & 95 \\
\hline $\begin{array}{l}\text { t.month.jan } \\
\text { (feb-nov) }\end{array}$ & dummy variable for a month when a presentation occurred. & 11 \\
\hline $\begin{array}{l}\text { t.wday.mon } \\
\text { (tue-sat) }\end{array}$ & dummy variable for a day of the week when a presentation occurred. & 6 \\
\hline holiday & $\begin{array}{l}\text { dummy variable specifying whether an ED presentation occurred on a public } \\
\text { holiday or one day before or after the holiday }\end{array}$ & 1 \\
\hline \multicolumn{3}{|c|}{ Patient-specific and other variables $(P)$} \\
\hline triage3 (4) & dummy variable specifying the triage status of a patient. & 2 \\
\hline $\mathrm{mh}$ & $\begin{array}{l}\text { dummy variable specifying whether a patient's presentation is mental-health } \\
\text { related }\end{array}$ & 1 \\
\hline ambu & $\begin{array}{l}\text { dummy variable specifying whether a patient arrived by ambulance or police } \\
\text { transport }\end{array}$ & 1 \\
\hline \multicolumn{3}{|c|}{ Weather $(W)$} \\
\hline temp & $\begin{array}{l}\text { average temperature in Brisbane recorded at the preceding 3-hour time interval } \\
\text { to the interval when a ED presentation was registered }\end{array}$ & 1 \\
\hline pressure & $\begin{array}{l}\text { atmospheric pressure at the weather station recorded at the preceding } 3 \text {-hour } \\
\text { time interval to the interval when a ED presentation was registered }\end{array}$ & 1 \\
\hline humid & $\begin{array}{l}\text { humidity recorded at the preceding 3-hour time interval to the interval when } \\
\text { a ED presentation was registered }\end{array}$ & 1 \\
\hline rain & $\begin{array}{l}\text { dummy variable specifying whether precipitation recorded at the preceding } \\
\text { 3-hour time interval to the interval when a ED presentation was registered }\end{array}$ & 1 \\
\hline \multicolumn{2}{|c|}{ Total number of predictors } & 140 \\
\hline
\end{tabular}


time. The disadvantage of estimating quantile regression at high quantile values is the increasing MSPE. This is clearly visible on the left graph in Figure 8 with rapidly increasing MSPE for quantiles above 0.7. For our analysis, we select 0.7 quantile for quantile regression, which provides comparable values for MSPE and percentage of underpredicted observations across the estimators.

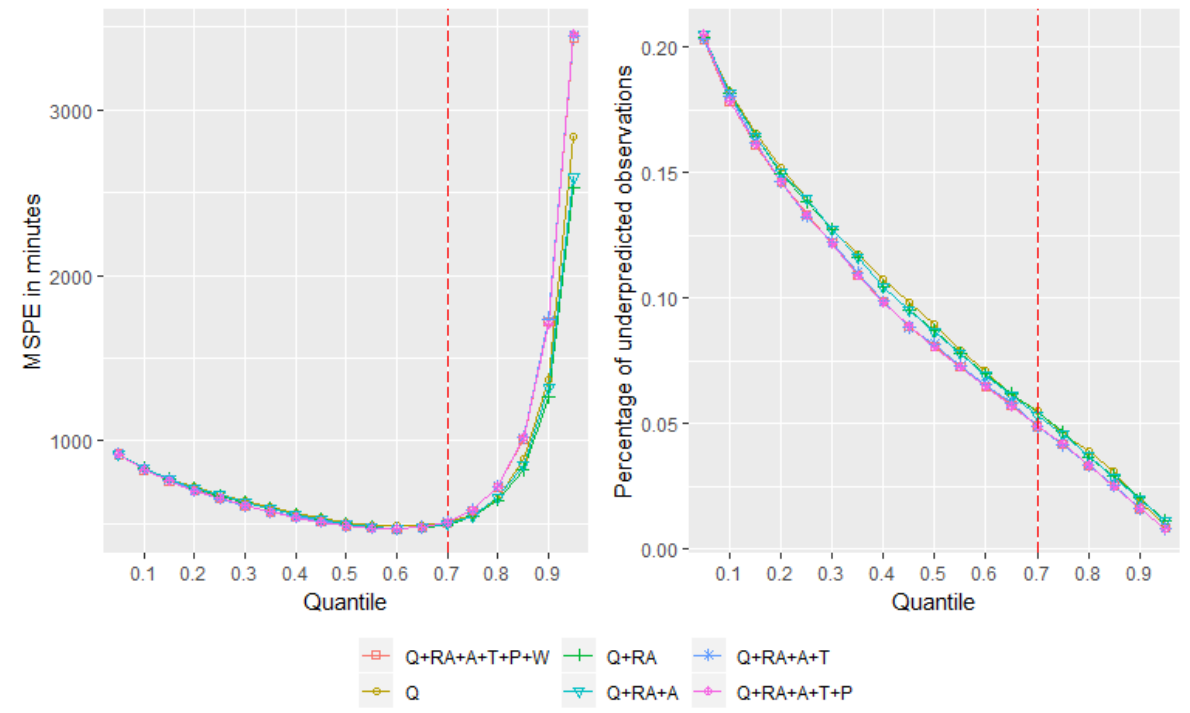

Figure 8: Quantile selection for quantile regression

Ridge, LASSO, OLS, quantile regression, and random forest predictive models and their specifications report higher accuracy than the commonly implemented rolling average method. The results in Table 3 show the importance of not only the model selection, but also what sets of predictors are used in the models. In general, ridge, LASSO, and OLS models perform similarly well across different specifications, but marginally LASSO achieves better accuracy than OLS and ridge based on our three criteria for model selection. Furthermore, MAPE criteria shows that LASSO consistently outperforms all other estimators (including quantile regression, random forest, and best rolling average). Although the quantile regression is inferior to LASSO based on MSPE, it consistently outperforms all estimators based on the percentage of underpredicted observations.

In Table 3 we compare the performance of models based on the different set of predictors. Specification 1 includes all predictors and acts as a reference point for the analysis. Under this specification LASSO provides slightly better results based on MSPE than ridge, OLS, quantile regression, and random forest but significantly outperforms 
$(>21 \%)$ the best rolling average prediction. The quantile regression generates predictions with $17 \%$ smaller MSPE and achieves the largest decrease (over 40\%) in the percentage of underpredicted observations when compared with the best rolling average forecasts. The results for specification 2 provide evidence that queueing and service flow predictors play a critical role in the prediction accuracy. Although the models contain only $\mathrm{Q}$ variables, the relative change in three criteria for ridge, LASSO, and OLS models is only around 1\% when compared to the full model. The differences between specifications 1 and 2 are more significant for random forest and quantile regression with clear preference for the larger set of relevant predictors based on MSPE and the percentage of undepredicted observations. The inclusion of the rolling average patient counts and waiting times (see specification 3) significantly improves the MSPE of random forest and quantile regression, but has a conflicting effect on the LASSO's criteria. Specifically, while LASSO's MSPE declines by $12 \mathrm{~min}$, there is an increase by 0.1 in the percentage of underpredicted observations. On the other hand, adding ambulatory care unit dummy to the set of predictors in specification 4 helps reduce the percentage of underpredicted observations by $0.1-0.2$ across the estimators. Accounting for the time and day of ED presentation (specification 5) slightly worsens the results with respect to MSPE for OLS, ridge, LASSO, and quantile regressions, but also helps decrease the percentage of underpredicted observations for quantile regression from 5.3 to 4.9. Overall, we treat specification 6 as our main set of predictors for predictive models as it provides marginally better or very similar results using three selection criteria across all specifications. From the practical perspective of implementing forecasting models in an emergency department in real-time, it is also preferable to use specification 6 rather than specification 1, as the weather data used in specification 1 needs to be sourced outside of the ED information system.

Quantile regression, random forest, and LASSO (together with ridge and OLS) deliver more accurate predictions than the best rolling average largely due to the underpredicted ED cases. Using queueing and service flow variables together with information on diurnal fluctuations helps statistical learning methods to reduce the number and the magnitude of underpredicted waiting times. Table 4 shows the quantile values for the error defined as the difference between the model predicted and actual waiting time. The rolling average method tends to underestimate the waiting time more than other methods 
Table 3: Model comparison for waiting room patients

\begin{tabular}{lccccc}
\hline Predictors & Ridge & LASSO & OLS & Random Forest & Quantile \\
\hline 1. Q + RA + A + T + P + W & $483(6.1)$ & $482(6.1)^{*}$ & $486(6.1)$ & $489(6.0)$ & $508(4.9)$ \\
2. Q & $487(6.3)$ & $487(6.2)^{*}$ & $493(6.1)$ & $534(6.2)$ & $507(5.5)$ \\
3. Q + RA & $475(6.3)$ & $475(6.3)^{*}$ & $478(6.2)$ & $491(6.1)$ & $495(5.4)$ \\
4. Q + RA + A & $477(6.1)$ & $477(6.2)^{*}$ & $481(6.0)$ & $495(6.0)$ & $499(5.3)$ \\
5. Q + RA + A + T & $483(6.1)$ & $482(6.1)^{*}$ & $487(6.0)$ & $489(6.1)$ & $511(4.9)$ \\
6. Q + RA + A + T + P & $482(6.0)$ & $481(6.0)^{*}$ & $486(6.0)$ & $488(6.1)$ & $509(4.9)$ \\
\hline
\end{tabular}

Notes: For the description of predictors see Table 2. We report three criteria for model selection: 1) MSPE in minutes; 2) in parenthesis, the percentage of observations in the test sample that were underpredicted by more than 30 minutes; 3$)^{*}$ denotes the best model for a particular set of predictors based on MAPE. We also compute the prediction error for 3-hour rolling average method (the best model among 1-6 hour rolling averages): 1) MSPE: 611;2) 8.5\%; 3) MAPE: 15.1, which is consistently inferior to quantile regression, LASSO, ridge, OLS, and random forest. Testing sample size $=7859$.

leading to the higher MSPE and MAPE values. If we consider that underprediction is a more serious concern than overprediction, the rolling average estimator is also inferior to other methods as shown in the column for the median quantile in Table 4. In reality, the problem of underpredicting waiting time by rolling average may be even more severe, as some hospital EDs include in the rolling average calculation the waiting time of high acuity patients, which is often minimal. In addition, the rolling average method tends to overestimate when the waiting times are short and underestimate when the waiting times are long because of the influence of diurnal fluctuations on the waiting time.

Table 4: Error quantile (Predicted - Actual time-to-treatment) using testing sample

\begin{tabular}{lccccc}
\hline Prediction Model & $\mathbf{5 \%}$ & $\mathbf{2 5 \%}$ & $\mathbf{5 0 \%}$ & $\mathbf{7 5 \%}$ & $\mathbf{9 5 \%}$ \\
\hline Ridge & -33.81 & -6.03 & 4.36 & 12.76 & 27.43 \\
LASSO & -33.90 & -6.01 & 4.28 & 12.60 & 27.39 \\
OLS & -33.54 & -5.97 & 4.43 & 13.11 & 28.37 \\
Random Forest & -33.88 & -4.72 & 6.12 & 13.72 & 26.21 \\
Quantile & -29.68 & -2.27 & 7.96 & 16.63 & 31.72 \\
Best Rolling Average & -39.80 & -11.14 & -0.10 & 8.45 & 25.37 \\
\hline
\end{tabular}

Notes: errors are in minutes. Testing sample size $=7859$

In Figure 9 we present more detailed results for the performance of the models based on the percentage of underpredicted observations. While MSPE and MAPE evaluate the average size of the prediction error, the selection criterion of the percentage of underpredicted observations minimises the effect of ED cases with large prediction errors and helps investigate the rate at which the number of undepredictions declines. Ang et al. (2015) report some evidence that patients are more dissatisfied when not treated after the predicted waiting time elapsed forgetting that the waiting time prediction is only in- 
dicative and exhibit a non-binding commitment. Overall, quantile regression consistently outperforms all other models and has a smaller number of underpredicted cases, especially when the waiting time undepredictions in minutes are relatively small (up to 15 minutes). In Table 3 we report the percentage of observations in the testing sample that were underpredicted by more than 30 minutes, and the percentage for quantile regression is 1.1 percentage points smaller than LASSO's criteria and 3.6 percentage points smaller than rolling average's one. What is also noticeable is that all our estimators significantly outperform the rolling average, by $30 \%$ or more in relative terms.

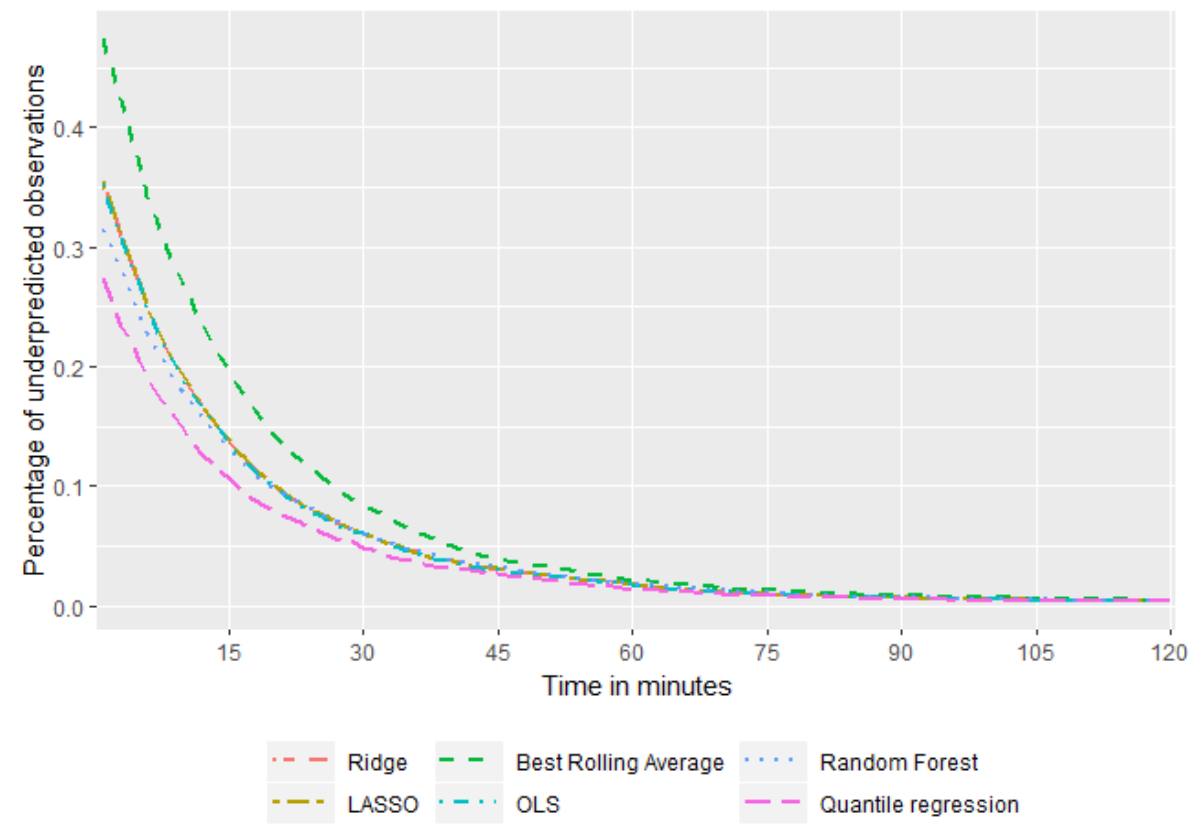

Figure 9: Percentage of underpredicted waiting time

The effect of diurnal fluctuations in the waiting times on performance of predictive models is presented in Figure 10. Compared to the actual waiting time for the testing sample, the best rolling average performs poorly and underestimates the waiting time during the day, except for the period when the actual average waiting time is at the lowest levels from around 6 am to $10 \mathrm{am}$. On the other hand, ridge, OLS, and LASSO tend to overpredict the waiting time more, but the magnitude of average overpredictions varies substantially during the day. By selecting above median quantile $\left(70^{\text {th }}\right)$ the quantile regression tends to consistently overpredict the actual waiting time during the day, but this comes at a cost of larger prediction errors in comparison to LASSO or random forest. The predictive models generate more accurate predictions when the ED operates at a 
dedicated clinical team for this patient cohort during the daytime (9 am $-9 \mathrm{pm})$ meaning that the demand for ED services and service rates are more predictive, and dedicated team at $\mathrm{ACU}$ treats low acuity patients. This helps to reduce the variance of the estimates and produce more accurate predictions. Figure 10 shows that random forest estimates are also closer to the actual waiting time during the daytime period and the variation of the average predicted waiting time from the actual waiting time during the day is relatively small, except for the period between 6 am and $11 \mathrm{am}$, when the arrivals of patients start to increase for the day.

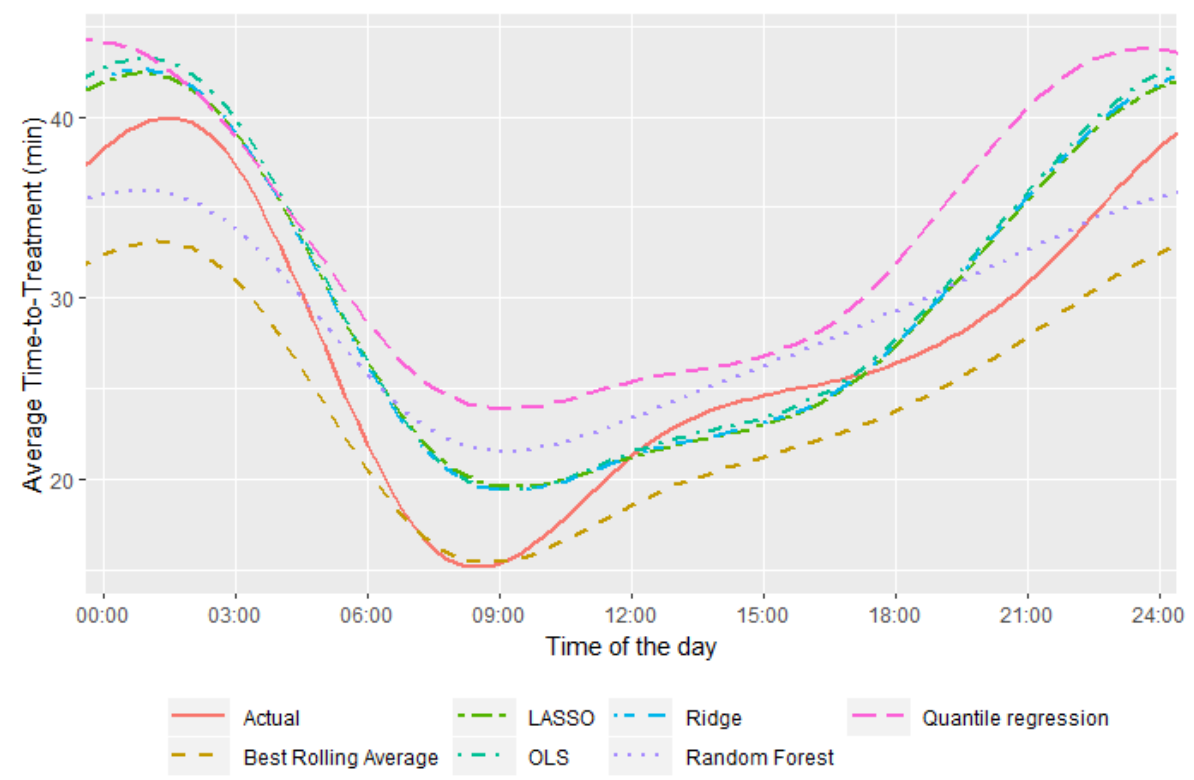

Figure 10: Average actual and predicted time-to-treatment for waiting room patients

Absolute deviations between the predicted and actual waiting time compared to the actual waiting times are presented in Figure 11. On average, all our estimators perform well at predicting waiting times for the waiting room patients with the actual waiting time up to 50 minutes. For these patients the average deviation between predicted and actual waiting time is relatively consistent and on average is around 11.75 minutes. From Figure 12 we see that approximately $90 \%$ of the waiting room patients do not wait more than 50 minutes, while LASSO and quantile regression predict that $96 \%$ and $93 \%$ of patients respectively would not need to wait for more than 50 minutes. From Figure 11 it is also clear that the prediction error increases rapidly as the waiting time becomes higher. Relative to a waiting room patient with an actual waiting time of between 50 to 60 minutes who experience the 


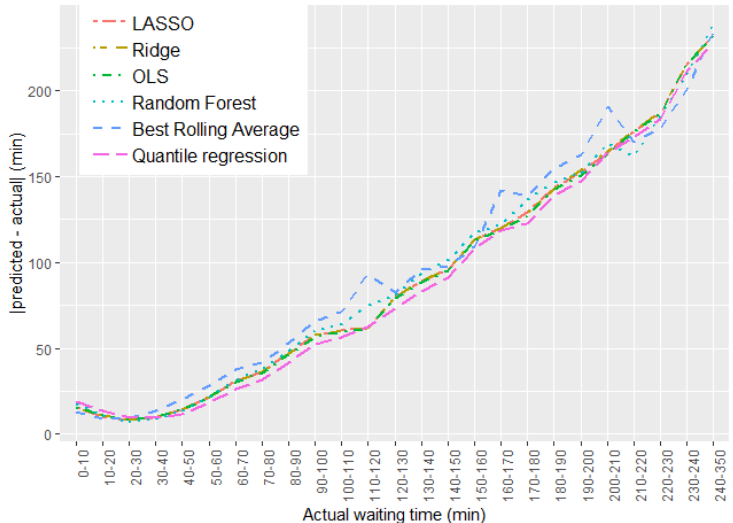

Figure 11: Variation between predicted and actual waiting time

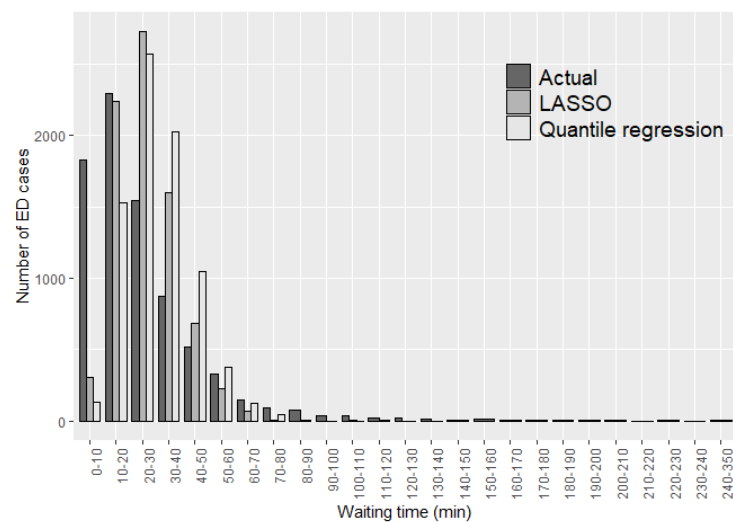

Figure 12: Number of ED presentations by actual waiting time

prediction error of approximately 22 minutes on average, a patient who waits between 60 and 70 minutes would get a prediction error of 31 minutes. This dependence is not particularly surprising as the number of patients experiencing long waiting time is small, hence the information used in the statistical learning algorithms is not sufficient to produce more accurate results leading to large prediction errors. For example, while the number of waiting room patients who waited more than 90 minutes in the testing sample is 168 out of 7,859 patients, LASSO and quantile regression significantly underpredict the waiting time for these patients and provide the waiting time predictions with over 90 minutes to only 6 and 8 patients respectively. Ideally, it would be beneficial to separately predict and manage patients who will have very prolonged wait times, thus enabling more accurate prediction of wait times for the majority of low acuity patients. Although the task is non-trivial because we are unable to know a priori whether a patient will experience a long waiting time, there is a potential to estimate the probability whether a patient is more likely to experience longer waiting time using patient-specific characteristics, time variables and ED busyness factors.

Following Figure 10, which provides initial evidence of the effect of diurnal fluctuations on the accuracy of the predictive models, we generate the estimates for the prediction errors in Table 5 using only patients who were registered in the ED from 9 am to $9 \mathrm{pm}$. Besides the fact that LASSO, ridge, OLS, and random forest resemble well the pattern of the actual waiting time in Figure 10, we select this time period because the majority of the patients arrive and get treated during this period. As expected, the results across all 
models presented in Table 5 report higher accuracy when compared to the ones in Table 3. This is most likely due to the lower variance of the predictors and the operation of the ACU meaning that low acuity patients have their dedicated team of nurses and clinicians to treat them and do not need to compete for staff, bed, and other resources with more urgent patients. Relative to the best MSPE performing model for the full testing sample (LASSO model, specification 6 in Table 3), LASSO regression with only queueing and service flow predictors (specification 2 in Table 5) reduces the MSPE by $30 \%$ and has the percentage of underpredicted observations declined from $6.0 \%$ to $4.6 \%$. On the other hand, while the MSPE for the quantile regression improved by more than $20 \%$, the percentage of underpredicted observations only decreased from $4.9 \%$ in the full testing sample to $4.4 \%$ in the restricted one. The results from Table 5 provide an insight of the importance of queueing and service variables for all estimators and the limited improvement in the results from other variables for LASSO, ridge, and OLS. This shows that at least for some predictive models using simpler specifications may be preferable. Importantly, the results for the quantile regression support specification 6 (as the results for the full sample), which includes all predictors except the weather ones.

Table 5: Model comparison for waiting room patients registered between 9 am and $9 \mathrm{pm}$

\begin{tabular}{lccccc}
\hline Predictors & Ridge & LASSO & OLS & Random Forest & Quantile \\
\hline Q + RA + A + T + P + W & $328(5.3)$ & $327(5.2)^{*}$ & $331(5.2)$ & $340(5.0)$ & $394(4.4)$ \\
Q & $339(4.7)$ & $339(4.6)^{*}$ & $347(4.5)$ & $385(4.6)$ & $392(4.7)$ \\
Q + RA & $325(4.8)$ & $325(4.8)^{*}$ & $330(4.7)$ & $346(4.8)$ & $386(4.6)$ \\
Q + RA + A & $322(4.8)$ & $322(4.8)^{*}$ & $327(4.7)$ & $343(4.7)$ & $386(4.6)$ \\
Q + RA + A + T & $328(5.3)$ & $328(5.2)^{*}$ & $332(5.2)$ & $340(5.0)$ & $395(4.4)$ \\
Q + RA + A + T + P & $328(5.2)$ & $327(5.2)^{*}$ & $331(5.2)$ & $338(5.0)$ & $393(4.4)$ \\
\hline
\end{tabular}

Notes: We report three criteria for model selection: 1) MSPE in minutes; 2) in parenthesis, the percentage of observations in the test sample that were underpredicted by more than 30 minutes; 3) * denotes the best model for a particular set of predictors based on MAPE. We also compute the prediction error for 3-hour rolling average method (best model among 1-6 hour rolling averages): 1) MSPE: 392; 2$) 7.4 \%$; 3) MAPE: 13.1, which is inferior to quantile regression, LASSO, ridge, OLS, and random forest. Testing sample size $=6055$.

With respect to the best rolling average, the quantile regression does not improve on MSPE criteria but has significantly lower percentage of underpredicted observations, $4.4 \%$ against $7.4 \%$. LASSO performs better in both criteria than the rolling average. In the constrained testing sample ( $9 \mathrm{am}-9 \mathrm{pm})$ there are $4.6 \%$ of patients who experienced the waiting time of 30 minutes or more than their predicted waiting time estimated by the best LASSO, ridge, OLS or random forest. On the other hand, using the rolling 
average estimator increases this number to $7.4 \%$, which is not largely different from the $8.5 \%$ in the full testing sample. This again shows that the rolling average method has low prediction accuracy and unable to account for the diurnal variations in the waiting times. These results are useful from the practitioner's perspective. If a hospital ED targets to minimise the number of patients with large underpredictions that potentially cause patient dissatisfaction, it can restrict the provision of waiting time predictions to patients that arrive when ED operates at a dedicated clinical team for this patient cohort.

\section{Conclusion}

Using more than 122,000 ED presentations from a large tertiary public ED, we used multiple statistical learning algorithms (LASSO, ridge, and random forest) and quantile regression methods to generate waiting time predictions for low acuity patients assigned to the ED waiting room. Relative to the commonly implemented rolling average, which only requires historical information for estimation, LASSO, ridge, OLS, quantile regression, and random forest estimators use a large set of generated predictors accounting for ED complex and data-rich dynamic environment. We find robust evidence that these five estimators significantly outperform the rolling average method with respect to the percentage of underpredicted observations, MSPE, and MAPE criteria. Our findings suggest that LASSO regression has the smallest MSPE and MAPE among models and across different specifications. Using queueing and service flow variables together with information on diurnal fluctuations LASSO delivers more accurate predictions than the best rolling average and reduce the number and the magnitude of underpredicted and overpredicted waiting times. Furthermore, the quantile regression estimator performs much better if the estimators are compared on the percentage of underpredicted observations. While the estimator has $5 \%$ larger MSPE than the best LASSO results, the quantile regression outperform the LASSO estimator in the percentage of underpredicted observations by almost $20 \%$ in relative terms. The results of quantile regression depend on the quantile selected for the estimator, as higher quantile values place higher weights on the positive residuals resulting in the lower number of underpredicted observations. But the lower number of underpredictions is achieved at the cost of higher number and magnitude of 
overpredictions. Depending on the trade-off between waiting time predictive accuracy and the percentage of underpredicted observations, hospital ED may prefer either LASSO or quantile regression for generating waiting time forecasts for the low acuity ED patients. To increase the predictive accuracy and minimise the number of patients with large underpredictions which potentially cause patient dissatisfaction, a hospital ED may decide to provide predictions to patients registered only during the daytime (9 am - $9 \mathrm{pm})$ when the ED operates at full capacity translating to more predictive service rates and the demand for treatments.

The results from our paper have significant practical implications for health departments and hospitals. Improving the accuracy of prediction estimates is critical to delivering waiting time forecasts to patients. By implementing the proposed estimators and reporting more accurate ED waiting times, individual hospitals may enjoy higher patient satisfaction and reduction in the likelihood of patients leaving without being seen by a medical professional. Larger benefits can also be achieved if multiple nearby hospitals publish their real-time waiting time estimates online for prospective low acuity patients. Due to this network coordination effects with low acuity patients selecting EDs on the basis of waiting time and travel distance, hospital EDs may have smoother distribution of patient loads among health providers.

Our theoretical framework of queue development and demand for services is validated by our results. By providing patients with accurate waiting time predictions $(t)$ at the triage stage, hospitals have better ability to identify patients who join or abandon the queue to receive treatment. 


\section{References}

American College of Emergency Physicians (2012). Publishing wait times for emergency department care: An information paper. https://www.acep.org/globalassets/ uploads/uploaded-files/acep/clinical-and-practice-management/resources/ crowding/wait_times_ip_june_2012.pdf. Accessed: 2018-07-01.

Ang, E., Kwasnick, S., Bayati, M., Plambeck, E. L., and Aratow, M. (2015). Accurate emergency department wait time prediction. Manufacturing $\&$ Service Operations Management, 18(1):141-156.

Batt, R. J. and Terwiesch, C. (2015). Waiting patiently: An empirical study of queue abandonment in an emergency department. Management Science, 61(1):39-59.

Boudreaux, E. D. and O'Hea, E. L. (2004). Patient satisfaction in the emergency department: A review of the literature and implications for practice. The Journal of Emergency Medicine, 26(1):13 - 26.

Bruni, M. L., Mammi, I., and Ugolini, C. (2016). Does the extension of primary care practice opening hours reduce the use of emergency services? Journal of Health Economics, $50: 144-155$.

Ding, Y., Park, E., Nagarajan, M., and Grafstein, E. (2019). Patient prioritization in emergency department triage systems: An empirical study of the Canadian triage and acuity scale (CTAS). Manufacturing \& Service Operations Management, 21(4):723-741.

Dolton, P. and Pathania, V. (2016). Can increased primary care access reduce demand for emergency care? Evidence from England's 7-day GP opening. Journal of Health Economics, 49:193 - 208 .

Dong, J., Yom-Tov, E., and Yom-Tov, G. B. (2018). The impact of delay announcements on hospital network coordination and waiting times. Management Science.

Hastie, T., Tibshirani, R., and Friedman, J. H. (2009). The elements of statistical learning: data mining, inference, and prediction. Springer, 2 edition. 
Hemaya, S. A. K. and Locker, T. E. (2012). How accurate are predicted waiting times, determined upon a patient's arrival in the emergency department? Emergency Medicine Journal, 29(4):316-318.

Mowen, J. C., Licata, J. W., and McPhail, J. (1993). Waiting in the emergency room: How to improve patient satisfaction. Journal of Health Care Marketing, 13(2):26.

Queensland Audit Office (2014). Emergency department performance reporting (Report 3: 2014-2015). https://www.qao.qld.gov.au/sites/qao/files/reports/rtp_ emergency_department_performance_reporting.pdf. Accessed: 2017-05-01.

Soremekun, O. A., Takayesu, J. K., and Bohan, S. J. (2011). Framework for analyzing wait times and other factors that impact patient satisfaction in the emergency department. The Journal of Emergency Medicine, 41(6):686 - 692.

Sullivan, C., Staib, A., Ayre, S., Daly, M., Collins, R., Draheim, M., and Ashby, R. (2016). Pioneering digital disruption: Australia's first integrated digital tertiary hospital. Medical Journal of Australia, 205(9):386-389.

Sun, Y., Teow, K. L., Heng, B. H., Ooi, C. K., and Tay, S. Y. (2012). Real-time prediction of waiting time in the emergency department, using quantile regression. Annals of Emergency Medicine, 60(3):299 - 308.

Thompson, D. A., Yarnold, P. R., Williams, D. R., and Adams, S. L. (1996). Effects of actual waiting time, perceived waiting time, information delivery, and expressive quality on patient satisfaction in the emergency department. Annals of Emergency Medicine, $28(6): 657-665$.

Weiner, S. G. (2013). Advertising emergency department wait times. Western Journal of Emergency Medicine, 14(2):77.

Xu, K. and Chan, C. W. (2016). Using future information to reduce waiting times in the emergency department via diversion. Manufacturing 83 Service Operations Management, 18(3):314-331. 